\title{
Total oesophago-gastric dissociation in neurologically impaired children: Laparoscopic vs robotic approach
}

\author{
Girolamo Mattioli $^{1,2}$ | Michela C.Y. Wong ${ }^{1,2}$ | Rossella Angotti ${ }^{3}$ | Cinzia Mazzola ${ }^{2}$ | \\ Serena Arrigo $^{4}$ | Paolo Gandullia ${ }^{4}$ | Margherita Mancardi ${ }^{5}$ | Giulia Fusi ${ }^{3}$ | \\ Mario Messina $^{3}$ | Clelia Zanaboni ${ }^{6}$ | Barbara Razore ${ }^{2}$ | Arrigo Barabino ${ }^{4}$ | \\ Francesco Molinaro ${ }^{3}$
}

${ }^{1}$ DINOGMI, University of Genoa, Genoa, Italy

${ }^{2}$ Paediatric Surgery Unit, Giannina Gaslini Children's Hospital, Institute for Scientific Research, Genoa, Italy

${ }^{3}$ Department of Medical Sciences, Surgery and Neuroscience, Section of Pediatric Surgery, University of Siena, Siena, Italy

${ }^{4}$ Pediatric Gastroenterology Unit, Giannina Gaslini Children's Hospital, Institute for Scientific Research, Genoa, Italy

${ }^{5}$ Pediatric Neuromuscular Unit, Giannina Gaslini Children's Hospital, Institute for Scientific Research, Genoa, Italy

${ }^{6}$ Department of Pediatric Anesthesia, Giannina Gaslini Children's Hospital, Institute for Scientific Research, Genoa, Italy

\section{Correspondence}

Francesco Molinaro, Department of Medical Sciences, Surgery and Neuroscience, Section of Pediatric Surgery, University of Siena, Policlinico Le Scotte, Viale Bracci 14, 53100 Siena, Italy.

Email: fmolidoc@me.com

\begin{abstract}
Purpose: To evaluate and compare the feasibility and short-term results of laparoscopic and robotic total oesophago-gastric dissociation (TOGD) with a Roux-en-Y oesophago-jejunostomy. Minimal invasive surgery has multiple advantages in neurologically impaired patients. Robotic approach has overcome disadvantages linked to laparoscopy, in particular, referring to the surgeon fatigue.

Methods: A retrospective study comparing five laparoscopic and five robotic TOGD was conducted between February and October 2016 in Giannina Gaslini Children's Hospital and Section of Pediatric Surgery of Siena. Neurologically impaired children scheduled for TOGD were included. Age, sex, weight, symptomatology, presence of epilepsy, and preoperative $\mathrm{X}$-ray contrast were considered. Operative time, hospital stay, postoperative complications, redo surgery, nutrition rehabilitation, and $\mathrm{X}$-ray contrast study after 5 days and after 1 month from the intervention were recorded.

Results: In our series, there were no intraoperative complications, no conversions to open surgery, and no vagal lesions. In two of five robotic cases, a pyloroplasty was necessary. The median operative time was statistically longer in the robotic group. One dehiscence in the robotic group was recorded, and no dumping episodes occurred. No statistical differences in terms of complications were detected.

Conclusion: TOGD is feasible both with laparoscopic and robotic-assisted surgery with similar results. Robotic approach is considered feasible. At the same time, high laparoscopic skills allow to reach the same results as robotic approach with shorter operative time.
\end{abstract}

\section{KEYWORDS}

gastro-oesophageal reflux, laparoscopic surgery, mininvasive surgery, neurological impairment, paediatric surgery, robotic surgery

\section{1 | INTRODUCTION}

Total oesophago-gastric dissociation (TOGD) with a Roux-en-Y oesophago-jejunostomy and a feeding gastrostomy is an anti-reflux procedure that was firstly described by Bianchi in 1997 as a "rescue" procedure for children with neurological or physical impairment. ${ }^{1}$ For children with gastro-oesophageal reflux (GOR) and a neurological impairment, especially for ones affected by seizures, the TOGD can 
be considered a valid alternative method, not only as a "rescue" intervention after fundoplication but also as first intervention choice. ${ }^{2-5}$ There are recent studies that underline the advantages of TOGD compared with the minimally invasive fundoplication techniques for the treatment of gastro-oesophageal reflux disease (GORD) in patients with severe neurological impairment. One of the disadvantages of this technique was the need, at the beginning, to be performed with an open approach, while the fundoplication has been developed, in the last two decades, as a laparoscopic procedure. Recent studies with a longer follow-up demonstrate in fact that the percentage of reflux relapse in patient with severe neurological impairment is high in patients treated with laparoscopic fundoplication (Molinaro et $\mathrm{al}^{2}$ ). The first laparoscopic TOGD in children was described in 2009 by Boubnova et al as a feasible procedure with evident advantages, especially in patients with severe neurological impairment, respiratory, and/or nutritional failure status. ${ }^{3}$ Mattioli et al described the first cases of robotic-assisted TOGD in $2017 .{ }^{4}$

The aim of our study is to present our experience and short-term results about TOGD in children and to evaluate the differences between laparoscopic and robotic approaches in terms of feasibility.

\section{2 | MATERIALS AND METHOD}

From February to October 2016, a retrospective study was conducted. In our cohort, we recruited for TOGD with a Roux-en-Y oesophago-jejunostomy and a feeding gastrostomy all neurologically impaired children with severe GOR refractory to medical treatment, associated to swallowing difficulties, recurrent ab ingestis pneumonitis, failure to thrive, and poor quality of life. The idea of the study stems from the fact that the intervention was designed and executed firstly with a robotic approach and then was tested for the feasibility of being performed also in laparoscopy. Because of the fact that a group of patients had already undergone a robotic procedure, it was not possible to devise a pre-study randomization protocol.

In order to distinguish patients with more severe neurological deficit, we used as a rating scale the Gross Motor Function Classification System Expanded and Revised (GMFCS-E\&R), which evaluates as parameters the following ones: ambulation, functional limitations, the need for devices for mobility or mobility in a wheelchair, and, to a lesser extent, the quality of the movement. It consists of five levels, of which the fifth (to be transported in a wheelchair) is what we used to distinguish patients with more severe neurological deficit. In addition to this assessment scale, we took into account the degree of feeding difficulties and breathing problems, carefully assessing their clinical condition. Informed consent was obtained from all patients.

All the patients were evaluated in the preoperative period with an X-ray contrast study, and a gastroenterological/surgical counselling was managed. The following items were considered: age, sex, weight, symptomatology (regurgitation/vomiting, poor weight gain, food refusal, and pneumonitis ab ingestis), presence of other respiratory diseases, and presence of epilepsy.
We included both children who had never undergone surgery and those who had undergone previous interventions of fundoplication. The intervention was performed with laparoscopic or robotic approach. Operative time, hospital stay, postoperative complications, redo surgery, $\mathrm{X}$-ray contrast study after 5 days and after 1 month from the intervention, and nutrition rehabilitation were recorded. Postoperative complications were classified by the application of the modified Clavien classification system.

Statistical analysis was performed using Wilcoxon signed rank test for paired data $(P<.05)$. In addition, the correlation between postoperative short-term complications was assessed using Fisher exact test $(P<.05)$.

Informed consent for enrolment in the study was obtained from the parent or caregiver of the patient. Written informed consent was obtained from the parent/caregiver of the patient for publication of the images.

The choice of this approach, both robotic and laparoscopic, is recent; therefore, the follow-up range is from 1 to 2 years.

\subsection{Surgical technique}

TOGD has been performed following the technique described by Adrian Bianchi. The patient was supine with the legs in neutral position, considering the basic dystonic posture and muscular contraction of each patient (Figure 1). For the robotic approach, we used a Da Vinci SI system with only three arms. Five trocars were used: one 12-mm transumbilical trocar for a $30^{\circ}$ laparoscopic camera and two $5-\mathrm{mm}$ (8 $\mathrm{mm}$ in robotic-assisted approach) trocars in the right and left hypochondriac regions. Two further 5- or 3-mm disposable accessory trocars were used for suction, traction, and for LigaSure device (LigaSure TM Maryland- Covidien Medtronic, MN, USA). By a laparoscopic intraabdominal exploration, a marker suture was placed at $40 \mathrm{~cm}$ distal to the duodenojejunal flexure. The jejunum was exteriorized through the umbilical port incision and divided and sealed with the LigaSure at the marker suture. A tension-free vascularized isoperistaltic Roux-en-Y jejunal loop was fashioned distally dividing the mesenteric vessels appropriately. Bowel continuity was established by end-to side jejunojejunostomy at 20 to $30 \mathrm{~cm}$ from the proximal end of the Roux-en-Y, and the exteriorized bowel was replaced into the abdomen. After that, in the robotic approach, the docking is performed. The next step was the identification and isolation of the abdominal portion of the oesophagus, preserving paraoesophageal neurovascular structures (Figure 2) and its fixation to the diaphragm with two nonabsorbable stitches to avoid oesophageal retraction up into the posterior mediastinum. The correct and good visualization of the abdominal nervous system allows, in both approaches, a safer preservation of the vagus nerves. Then, a transmesocolic window and an adequate retrogastric space were created. The jejunal loop was later introduced through the mesocolic window at the left of the left gastric pedicle. The oesophagus was sectioned from the stomach using a monopolar scissor or the LigaSure. The gastric fundus was closed, and the oesophago-jejunal anastomosis was undertaken with 


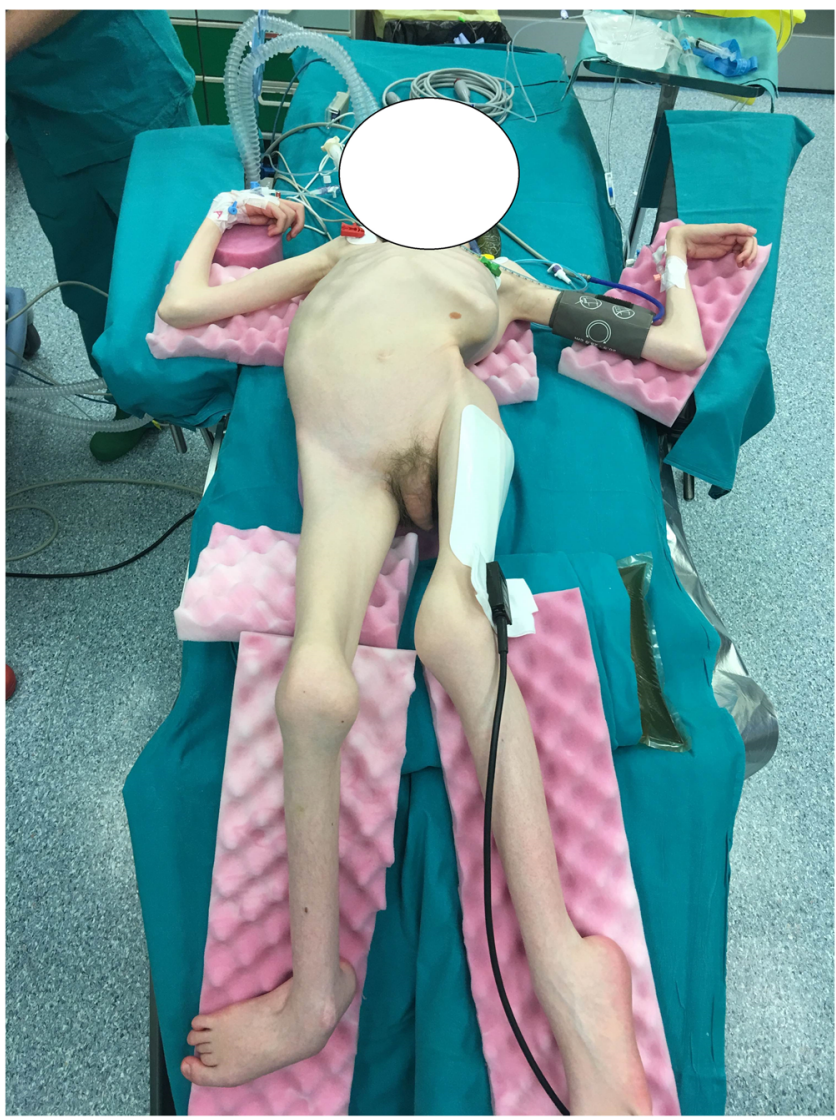

FIGURE 1 The patient position during the operation is altered by the dystonic posture and muscular contraction

4/0 interrupted sutures (Figures 3 and 4). The oesophago-jejunal anastomosis was performed in monofilament, first on the backside and then on the front. The suture on the gastric side was performed in vicryl. Once the oesophago-jejunal anastomosis was performed and the stomach was closed, the loop was fixed with a point to the mesocolic breach. Finally, a Stamm gastrostomy was done, and a pyloroplasty was associated if needed. The cases treated with pyloroplasty were those that showed a marked delay of gastric emptying at preoperative control.

\section{3 | RESULTS}

Data of 11 neurologically impaired children were evaluated in a retrospective analysis included for a TOGD. One girl $(4 \mathrm{~kg}, 3$ months of age) was excluded from the study because of the fragility and big dimensions of the liver. Ten TOGD were totally performed. Five of 10 (three boys and two girls) were operated with a laparoscopic approach and five of 10 (three boys and two girls) with a roboticassisted approach. In the laparoscopic group, the median weight and age were respectively $13 \mathrm{~kg}$ and 40 months, while in the robotic group, $12 \mathrm{~kg}$ and 48 months (no statistically differences, $P=.91$ ). Tables 1 and 2 describe the basic diseases and features of the patients in each group and the differences between the two groups regarding age, weight, and operative times, respectively.

Both in the laparoscopic group and in the robotic one, three of five children suffered from epilepsy.

Five of 10 patients had never undergone anti-reflux surgery before. In the laparoscopic group, only two patients of five had never undergone surgery before. The patient under the age of 1 year had already undergone surgery in another centre. This intervention led to a relapse of GOR due to mediastinal slippage of the fundoplication. One girl had undergone a Nissen fundoplication with gastrostomy and
FIGURE 2 The vagal nerve seen in the laparoscopic (on the left) and robotic (on the right) approaches
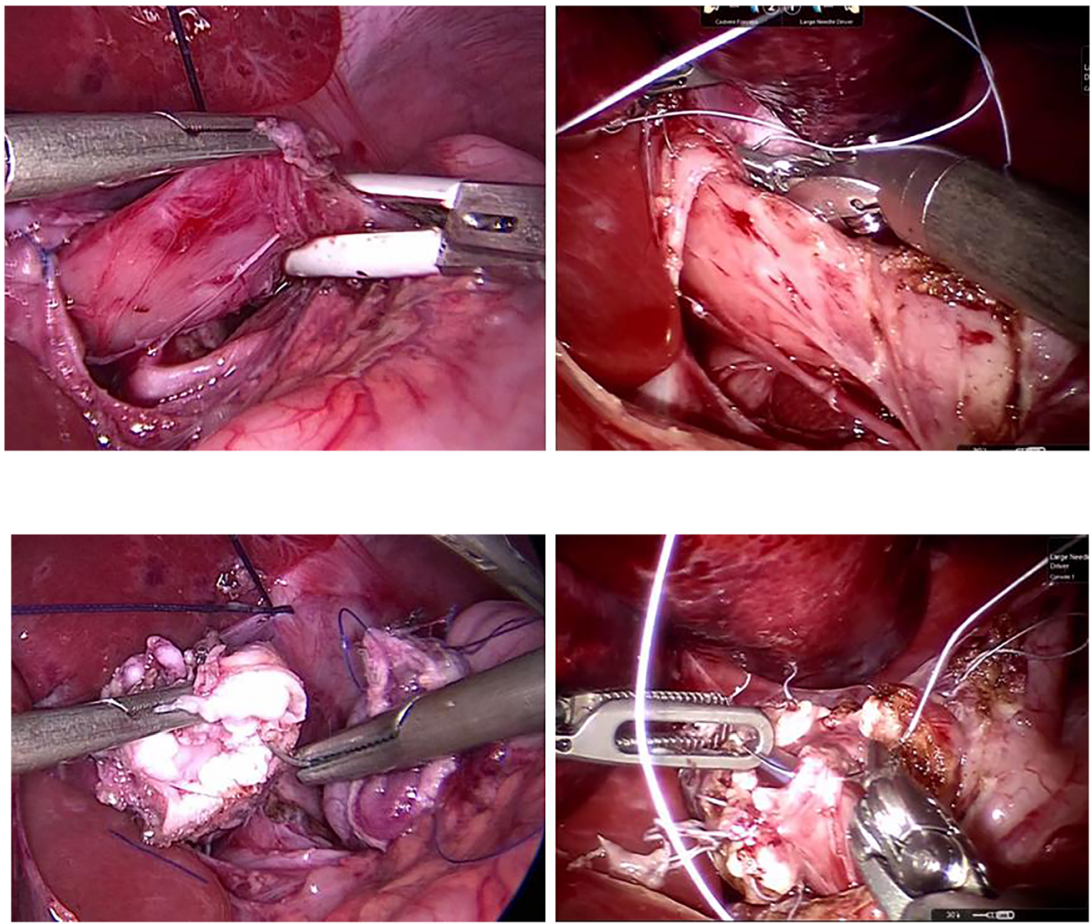

FIGURE 3 The beginning of the anastomosis seen in the laparoscopic (on the left) and robotic (on the right) approaches 

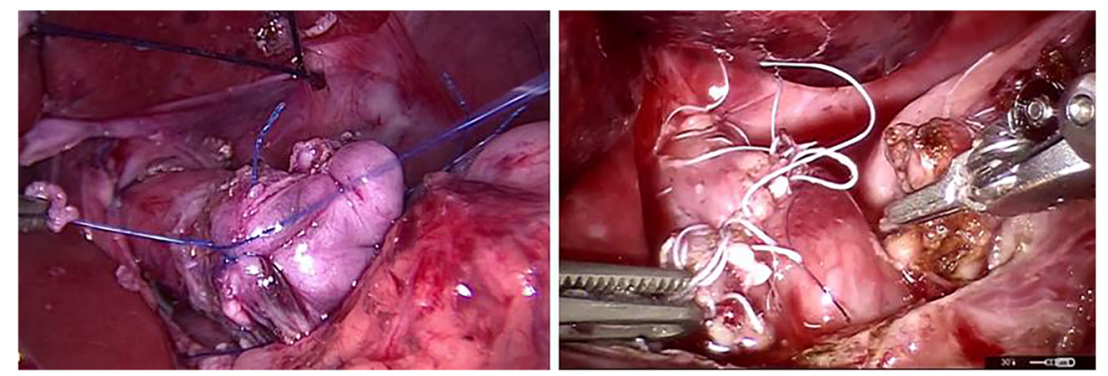

FIGURE 4 The end of the anastomosis seen in the laparoscopic (on the left) and robotic (on the right) approaches

TABLE 1 The basic diseases and features of the patients in robotic and laparoscopic group

\begin{tabular}{|c|c|c|c|}
\hline & Basic Disease & $\begin{array}{l}\text { Age, } \\
\text { mo }\end{array}$ & $\begin{array}{l}\text { Weight, } \\
\text { kg }\end{array}$ \\
\hline \multicolumn{4}{|c|}{ Robotic group } \\
\hline Patient 1 & $\begin{array}{l}\text { Infant cerebral palsy due to } \\
\text { neonatal hypoxia with a spastic } \\
\text { dystonic quadriparesis, carrier of } \\
\text { a ventriculo-peritoneal shunting } \\
\text { for congenital hydrocephalus }\end{array}$ & 9 & 6 \\
\hline Patient 2 & $\begin{array}{l}\text { Infant cerebral palsy due to } \\
\text { neonatal hypoxia with a spastic } \\
\text { dystonic quadriparesis }\end{array}$ & 108 & 17 \\
\hline Patient 3 & Lysosomal disease & 17 & 8 \\
\hline Patient 4 & VACTERL association & 40 & 13 \\
\hline Patient 5 & $\begin{array}{l}\text { Severe neurological impairment } \\
\text { and spastic quadriparesis due to } \\
\text { cardiac arrest at the age of } \\
16 \text { months }\end{array}$ & 171 & 30 \\
\hline \multicolumn{4}{|c|}{ Laparoscopic group } \\
\hline Patient 6 & $\begin{array}{l}\text { Infant cerebral palsy due to } \\
\text { neonatal hypoxia, oesophageal } \\
\text { atresia }\end{array}$ & 90 & 14 \\
\hline Patient 7 & $\begin{array}{l}\text { Infant cerebral palsy due to } \\
\text { neonatal hypoxia, carrier of an } \\
\text { intrathecal baclofen pump }\end{array}$ & 172 & 26 \\
\hline Patient 8 & $\begin{array}{l}\text { Infant cerebral palsy due to } \\
\text { neonatal hypoxia }\end{array}$ & 48 & 11 \\
\hline Patient 9 & $\begin{array}{l}\text { Drug-resistant epileptic } \\
\text { encephalopathy with severe } \\
\text { neuropsychological } \\
\text { deterioration }\end{array}$ & 20 & 8 \\
\hline Patient 10 & $\begin{array}{l}\text { Drug-resistant epileptic } \\
\text { encephalopathy with severe } \\
\text { neuropsychological } \\
\text { deterioration }\end{array}$ & 38 & 12 \\
\hline
\end{tabular}

a gastro-jejunal diversion (Roux en $\mathrm{Y}$ loop) 8 years before and a Nissen redo 1 year later. One boy had undergone a feeding gastrostomy 3 years before. In the robotic group, three of five children had never been operated on before. One boy had an intrathecal intraabdominal baclofen pump. One boy had undergone a laparoscopic Nissen fundoplication and a second procedure 1 year later with the placement of a diaphragmatic on-lay mesh, complicated by oesophageal perforation.
TABLE 2 The differences between the two groups regarding age, weight, and operative times

\begin{tabular}{llll} 
Group & $\begin{array}{l}\text { Median } \\
\text { Age, mo }\end{array}$ & $\begin{array}{l}\text { Median } \\
\text { Weight, kg }\end{array}$ & $\begin{array}{l}\text { Median Operative } \\
\text { Time, min }\end{array}$ \\
\hline Robotic approach & 40 & 13 & 290 \\
$\begin{array}{l}\text { Laparoscopic } \\
\text { approach }\end{array}$ & 48 & 12 & 170 \\
\hline P value & .91 & .91 & .004 \\
\hline
\end{tabular}

The median operative time of the laparoscopic and robotic TOGD was respectively 170 and 290 minutes. Wilcoxon signed-rank test of paired comparisons indicated that there is a significant difference between the two procedures about operative time $(P=.004)$. In two robotic cases, a pyloroplasty was done.

The median hospital stay was 17 days in the laparoscopic group and 18 days in the robotic one.

The X-ray contrast study on postoperative day (7 POD) did not show the presence of complications in all the children of the laparoscopic group and in four children of the robotic one. In the boy with oesophageal atresia history and oesophageal perforation in presence of an on-lay mesh, the X-ray contrast study detected a dehiscence of the oesophago-jejunal anastomosis.

In the laparoscopic group, the feeding restarted on POD 7 (range 5-11), while in the robotic group, on POD 8 (range 6-13). Feeding of the patients usually began with clear liquid feedings through the gastrostomy. The quantity of the feeding was gradually increased, both through the gastrostomy and orally.

Concerning the short-term complications, in the laparoscopic group, one patient had a left pleural effusion requiring the positioning of a tube drainage (grade IIlb of modified Clavien classification system). In the robotic group, one patient underwent oesophago-jejunal anastomotic dehiscence (grade IIlb of modified Clavien classification system). In the absence of infectious problems, we think it may depend on a technical problem. The patient currently does not prosecute any problems related to GORD. The leak was first treated conservatively, but later, a covered selfexpanding stent was placed with resolution of the leakage. Fisher exact test indicated that there is no significant difference between the two procedures about short-term complications $(P=1.00)$. No intraoperative complications were recorded, no conversions to open surgery were needed, and no vagal lesions nor dumping disorders were present. 
Finally the X-ray contrast study after 1 month from the operation did not detect complications in all the patients, except for the boy with multiple complications who still had the oesophago-jejunal leakage, and the decision of positioning the stent was taken after this exam (grade IIlb of modified Clavien classification system).

\section{DISCUSSION}

Fundoplication has always been considered the gold standard for the surgical treatment of GOR in patients refractory to conservative medical treatment. ${ }^{5}$ In neurologically impaired children, especially in ones affected by seizures, the risk of recurrence of GOR and mortality after a fundoplication is higher compared with patients with a normal neurological level. ${ }^{1,2,6-11}$ For this reason, in recent years, TOGD has obtained an higher consensus in the surgical treatment of GOR in this kind of patients. In fact, it is now considered as a valid option not only as a 'rescue' intervention in patients with recurrence of GOR after fundoplication, but also as primary procedure with good results. ${ }^{2,6,12-14}$ Our recent analysis of outcome has shown that the presence of seizures is a significant risk factors for surgical success of Nissen fundoplication. ${ }^{15}$

This study, comparing five laparoscopic TOGD and five robotic TOGD, was conducted after checking the feasibility of TOGD by robotics. In these patients, the use of a robotic approach is justified by the need to perform fine sutures. Moreover, the good advantages and results of this kind of surgery led us to consider the possibility of its feasibility also in laparoscopy. In fact, extending the minimally invasive approach even to a major intervention such as TOGD could bring benefits in terms of cost and advantages for these particular paediatric patients.

The initial approach by robotics, for the easiest realization of the oesophagus-jejunal anastomosis, has made clear the possibility of performing the intervention by minimally invasive, with an excellent visualization of the vague nerves. Driven by this awareness, we have achieved the same in laparoscopy. It is also feasible in laparoscopy, with lower costs, but it cannot be said that it is easier to carry out.

The median operative time was statistically longer in the robotic group. This reflects the longer docking time required with the robotic group. In our study, the learning curve for robotic approach and laparoscopic approach was similar. The laparoscopic TOGD and the robotic TOGD are similar in terms of feasibility, even if some differences are present. With the robotic approach, the first surgeon can work in a more comfortable position, confirming that robot-assisted surgery is less stressful than standard laparoscopy. ${ }^{16}$ This is true especially considering the kind of patients that underwent surgery. In fact, in children with important alterations of the basic posture with limb spasticity, robotic system can facilitate the staff and the patient position on the operating room bed.

Concerning the very short-term results, one oesophago-jejunal anastomotic dehiscence was detected in the robotic group. It is important to underline that this boy had a very complicated condition before the TOGD; in fact, he had an oesophageal atresia history and he had undergone two Nissen fundoplications and the positioning of an on-lay mesh some years before. Performing a TOGD in a patient that has already undergone one or two fundoplications or other abdominal interventions is technically more difficult and demanding than performing the same procedure as first intervention.

In conclusion, the TOGD is an anti-reflux intervention that can be performed both with a standard laparoscopic approach and a roboticassisted one with the same results. The surgical feasibility of the procedure is similar between the two approaches. The robotic surgery results to be more comfortable and easier for the surgeon especially considering the altered dystonic posture of these patients. At the same time, high laparoscopic skills give the possibility to perform the same procedure with shorter time and as good results as with the robotic approach.

\section{CONFLICT OF INTEREST}

Drs Girolamo Mattioli, Michela C.Y. Wong, Rossella Angotti, Cinzia Mazzola, Serena Arrigo, Paolo Gandullia, Margherita Mancardi, Mario Messina, Clelia Zanaboni, Barbara Razore, Arrigo Barabino, and Francesco Molinaro have no conflicts of interest or financial ties to disclose.

\section{ORCID}

Francesco Molinaro (D) https://orcid.org/0000-0002-8015-1233

\section{REFERENCES}

1. Bianchi A. Total esophagogastric dissociation: an alternative approach. J Pediatr Surg. 1997 Sep;32(9):1291-1294.

2. Molinaro F, Bindi E, et al. Esophagogastric dissociation reduces the re-operation rate for persistent gastroesophageal reflux in severely neurologically impaired children. Pediatr Surg Int. 2014 Oct;30(10): 997-1001.

3. Boubnova J, Hery G, Ughetto F, Charpentier A, Guys JM, de Lagausie P. Laparoscopic total esophagogastric dissociation. J Pediatr Surg. 2009 Oct;44(10):e1-e3.

4. Mattioli G, Molinaro F, Paraboschi I, et al. Robotic-assisted minimally invasive total esophagogastric dissociation for children with severe Neurodisability. J Laparoendosc Adv Surg Tech a. 2017 May;27(5): 550-555.

5. Horgan S, Pellegrini CA. Surgical treatment of gastroesophageal reflux disease. Surg Clin North am. 1997 Oct;77(5):1063-1082.

6. Islam S, Teitelbaum DH, et al. Esophagogastric separation for failed fundoplication in neurologically impaired children. J Pediatr Surg. 2004 Mar;39(3):287-291.

7. Morabito A, Lall A, et al. Total esophagogastric dissociation: 10 years' review. J Pediatr Surg. 2006 May;41(5):919-922.

8. Lansdale N, McNiff M, Morecroft J, Kauffmann L, Morabito A. Longterm and 'patient- reported' outcomes of total esophagogastric dissociation versus laparoscopic fundoplication for gastroesophageal reflux disease in the severely neurodisabled child. J Pediatr Surg. 2015 Nov; 50(11):1828-1832.

9. Peters RT, Goh YL, Veitch JM, Khalil BA, Morabito A. Morbidity and mortality in total esophagogastric dissociation: a systematic review. J Pediatr Surg. 2013 Apr;48(4):707-712.

10. Zaidi T, Sudall C, Kauffmann L, Folaranmi S, Khalil B, Morabito A. Physical outcome and quality of life after total esophagogastric dissociation in children with severeneurodisability and gastroesophageal reflux, from the caregiver's perspective. J Pediatr Surg. 2010 Sep;45 (9):1772-1776 
11. Zhang P, Tian J, et al. Laparoscopic vs. open Nissen's fundoplication for gastro-oesophageal reflux disease in children: a meta-analysis. Int J Surg. 2016 Oct;34:10-16.

12. Danielson PD, Emmens RW. Esophagogastric disconnection for gastroesophageal reflux in children with severe neurological impairment. J Pediatr Surg. 1999 Jan;34(1):84-86.

13. Goyal A, Khalil B, et al. Esophagogastric dissociation in the neurologically impaired: an alternative to fundoplication? J Pediatr Surg. 2005 Jun;40(6):915-918.

14. Gatti C, di Abriola GF, et al. Esophagogastric dissociation versus fundoplication: which is best for severely neurologically impaired children? J Pediatr Surg. 2001 May;36(5):677-680.

15. Rossi V, Mazzola C, et al. Long-term outcome and need of reoperation in gastro-esophageal reflux surgery in children. Pediatr Surg Int. 2016 Mar;32(3):277-283.
16. Van der Schatte Olivier RH, Van't Hullenaar CD, et al. Ergonomics, user comfort, and performance in standard and robot-assisted laparoscopic surgery. Surg Endosc. 2009 Jun;23(6):1365-1371.

How to cite this article: Mattioli G, Wong MCY, Angotti R, et al. Total oesophago-gastric dissociation in neurologically impaired children: Laparoscopic vs robotic approach. Int J Med Robotics Comput Assist Surg. 2019;e2048. https://doi.org/10. $\underline{1002 / \text { rcs.2048 }}$ 